\title{
Persistence and almost periodic solutions for a discrete ratio-dependent Leslie system with feedback control
}

\section{Zhouhong Li*}

\section{"Correspondence:} zhouhli@yeah.net

Department of Mathematics, Yuxi Normal University, Yuxi, Yunnan 653100, People's Republic of China

\begin{abstract}
In this paper, by utilizing the comparison theorem of the differential equation and constructing a suitable Lyapunov functional, we consider the existence of almost periodic solutions to a discrete time ratio-dependent Leslie system with feedback control. Some sufficient conditions for the existence of positive almost periodic solutions for the model are obtained. An example is given to illustrate the effectiveness of the main results.
\end{abstract}

MSC: 34K14; 92D25

Keywords: persistence; almost periodic solution; discrete ratio-dependent Leslie system; feedback controls

\section{Introduction}

In 1948, Leslie considered the following differential equation (see [1]):

$$
\left\{\begin{array}{l}
\dot{x}(t)=x(t)[a-b x(t)]-p(x) y(t), \\
\dot{y}(t)=y(t)\left[e-\frac{y(t)}{x(t)}\right]
\end{array}\right.
$$

where $x(t)$ and $y(t)$ stand for the population (the density) of the prey and the predator at time $t$, respectively, and $p(x)$ is the so-called predator functional response to prey.

Recently, more and more obvious evidences of biology and physiology show that in many conditions, especially when the predators have to search for food (consequently, have to share or compete for food), a more realistic and general predator-prey system should rely on the theory of ratio-dependence, this theory is confirmed by lots of experimental results (see [2, 3]). A ratio-dependent Leslie system with the functional response of HollingTanner type is as follows:

$$
\left\{\begin{array}{l}
\dot{x}(t)=x(t)[a-b x(t)]-p\left(\frac{x(t)}{y(t)}\right) y(t), \\
\dot{y}(t)=y(t)\left[e-\frac{y(t)}{x(t)}\right]
\end{array}\right.
$$

where $p(x)$ has the same means as before. In particular, Wang et al. [3] considered a ratiodependent Leslie predator-prey model with feedback controls as follows:

$$
\left\{\begin{array}{l}
\dot{x}_{1}(t)=x_{1}(t)\left[b(t)-a(t) x_{1}(t)-\frac{c(t) x_{1}(t) x_{2}(t)}{h^{2}(t) x_{2}^{2}(t)+x_{1}^{2}(t)}-d(t) u_{1}(t)\right], \\
\dot{x}_{2}(t)=x_{2}(t)\left[g(t)-f(t) \frac{x_{2}(t)}{x_{1}(t)}-p(t) u_{2}(t)\right], \\
\dot{u}_{i}(t)=\alpha_{i}(t)-\beta_{i}(t) u_{i}(t)+\gamma_{i}(t) x_{i}(t),
\end{array}\right.
$$


where $x_{1}(t)$ and $x_{2}(t)$ stand for the population (the density) of the prey and the predator at time $t$, respectively, $u_{i}(t)(i=1,2)$ are control variables, the prey grows logistically with growth rate $a(t)$ and carries capacity $\frac{a}{b}$ in the absence of predation. The parameter $f(t)$ is a measure of food quality that the prey provides, which is converted to the predator birth. Under the assumption that the coefficients of the above system are all $T$-periodic functions, by applying Mawhin's continuation theorem and constructing a suitable Lyapunov function, they obtained sufficient conditions which guarantee the existence of a unique globally attractive positive $T$-periodic solution to system (1.1).

Feedback control is the basic mechanism by which systems, whether mechanical, electrical, or biological, maintain their equilibrium or homeostasis. During the last decade, a series of mathematical systems have been established to describe the dynamics of feedback control systems, we refer to [4-9]. Furthermore, in recent research on species, dynamics of the Leslie system has important significance, see $[1-3,5,6,10-16]$ and the references therein for details. Moreover, since the discrete time models can also provide efficient computational models of continuous models for numerical simulations, it is reasonable to study discrete time models governed by difference equations. Motivated by the above idea, we consider a discrete ratio-dependent Leslie system with feedback control:

$$
\left\{\begin{array}{l}
x_{1}(n+1)=x_{1}(n) \exp \left\{b(n)-a(n) x_{1}(n)-\frac{c(n) x_{1}(n) x_{2}(n)}{h^{2}(n) x_{2}^{2}(n)+x_{1}^{2}(n)}-d(n) u_{1}(n)\right\} \\
x_{2}(n+1)=x_{2}(n) \exp \left\{g(n)-f(n) \frac{x_{2}(n)}{x_{1}(n)}-p(n) u_{2}(n)\right\} \\
\Delta u_{i}(n)=-\alpha_{i}(n) u_{i}(n)+\beta_{i}(n) x_{i}(n), \quad i=1,2
\end{array}\right.
$$

where $x_{i}(n)(i=1,2)$ denote the density of the prey and the predator at time $n$, respectively. $u_{i}(n), i=1,2$ are control variables, $b(n), a(n), c(n), d(n), g(n), f(n), p(n), h^{2}(n), \alpha_{i}(n), \beta_{i}(n)$, $\gamma_{i}(n)(i=1,2)$ are all almost $\omega$-periodic functions of $n ; h^{2}(n)$ denotes the constant of capturing half-saturation. For more biological background of system (1.2), one could refer to [3] and the references cited therein.

To the best of our knowledge, though many works have been done for population dynamic systems with feedback controls, most of the works deal with continuous time models. For more results about the existence of almost periodic solutions of a continuous time system, we can refer to [5] and the references cited therein. There are few works that consider the existence of almost periodic solutions for a discrete time population dynamic model with feedback controls. On the other hand, in fact, it is more realistic to consider almost periodic systems than periodic systems. On the existence and stability of almost periodic sequence solutions for the discrete biological models, some results are found in the literature, we refer to $[8,9,17,18]$. Therefore, our main purpose of this paper is to study the existence and uniqueness of almost periodic solutions for model (1.2).

Throughout this paper, we assume that

$\left(\mathrm{H}_{1}\right)\{a(n)\},\{b(n)\},\{c(n)\},\{d(n)\},\{h(n)\},\{g(n)\},\{f(n)\},\{p(n)\},\left\{\alpha_{i}(n)\right\}$ and $\left\{\beta_{i}(n)\right\}$ for $i=1,2$ are bounded nonnegative almost periodic sequences such that

$$
\begin{aligned}
& 0<a^{L}<a(n)<a^{M}, 0<b^{L}<b(n)<b^{M}, 0<c^{L}<c(n)<c^{M}, \\
& 0<d^{L}<d(n)<d^{M}, 0<h^{L}<h(n)<h^{M}, 0<g^{L}<g(n)<g^{M}, \\
& 0<p^{L}<p(n)<p^{M}, 0<f^{L}<f(n)<f^{M}, 0<\alpha_{i}^{L}<\alpha_{i}(n)<\alpha_{i}^{M}(i=1,2), \\
& 0<\beta_{i}^{L}<\beta_{i}(n)<\beta_{i}^{M}(i=1,2) .
\end{aligned}
$$

Here, for any bounded sequence $\{\theta(n)\}, \theta^{M}=\sup _{n \in N}\{\theta(n)\}$ and $\theta^{L}=\inf _{n \in N}\{\theta(n)\}$. Furthermore, we need the following assumptions: 
$\left(\mathrm{H}_{2}\right) g^{L}-p^{M} u_{2}^{*}>0$

$\left(\mathrm{H}_{3}\right) b^{L}-d^{M} u_{1}^{*}>0$.

By the biological meaning, we focus our discussion on the positive solution of model (1.2). So it is assumed that the initial conditions of model (1.2) are of the form

$$
x_{i}(0)>0, \quad u_{i}(0)>0, \quad i=1,2 .
$$

One can easily show that all the solutions of model (1.2) with the initial condition (1.3) are defined and remain positive for all $n \in \mathbb{Z}^{+}$.

The organization of this paper is as follows. In Section 2, we give some basic definitions and necessary lemmas which will be used in later sections. In Section 3, the persistence of model (1.2) is established. In Section 4, based on the persistence result, we show the existence and uniform asymptotic stability of an almost periodic solution to model (1.2). An example is given in Section 5.

\section{Definitions and lemmas}

Now let us state several definitions and lemmas which will be useful in proving the main result of this section.

Definition 2.1 [17] A sequence $x: \mathbb{Z} \rightarrow \mathbb{R}$ is called an almost periodic sequence if the $\epsilon$-translation number set of $x$,

$$
E\{\epsilon, x\}=\{\tau \in \mathbb{Z}:|x(n+\tau)-x(n)|<\epsilon, \forall n \in \mathbb{Z}\},
$$

is a relatively dense set in $\mathbb{Z}$ for all $\epsilon>0$; that is, for any given $\epsilon>0$, there exists an integer $l(\epsilon)>0$ such that each interval of length $l(\epsilon)$ contains an integer $\tau \in E\{\epsilon, x\}$ such that

$$
|x(n+\tau)-x(n)|<\epsilon, \quad \forall n \in \mathbb{Z},
$$

$\tau$ is called the $\epsilon$-translation number of $x(n)$.

Definition 2.2 [17] Let $f: \mathbb{Z} \times \mathbb{D} \rightarrow \mathbb{R}^{k}$, where $\mathbb{D}$ is an open set in $\mathbb{R}^{k}, f(n, x)$ is said to be almost periodic in $n$ uniformly for $x \in \mathbb{D}$, or uniformly almost periodic for short, if for any $\epsilon>0$ and any compact set $\mathbb{S}$ in $\mathbb{D}$, there exists a positive integer $l(\epsilon, \mathbb{S})$ such that any interval of length $l(\epsilon, \mathbb{S})$ contains an integer $\tau$ for which

$$
|f(n+\tau, x)-f(n, x)|<\epsilon, \quad \forall n \in \mathbb{Z}, x \in \mathbb{S},
$$

$\tau$ is called the $\epsilon$-translation number of $f(n, x)$.

Lemma 2.1 [18] $\{x(n)\}$ is an almost periodic sequence if and only iffor any sequence $\left\{h_{k}^{\prime}\right\} \subset$ $\mathbb{Z}$ there exists a subsequence $\left\{h_{k}\right\} \subset\left\{h_{k}^{\prime}\right\}$ such that $x\left(n+h_{k}\right)$ converges uniformly on $n \in \mathbb{Z}$ as $k \rightarrow \infty$. Furthermore, the limit sequence is also an almost periodic sequence.

In [17], Zhang and Zheng consider the following almost periodic delay difference system

$$
x(n+1)=f\left(n, x_{n}\right), \quad n \in \mathbf{Z}^{+},
$$


where $f: \mathbf{Z}^{+} \times C_{\mathbf{B}} \rightarrow \mathbf{R}, C_{\mathbf{B}}=\{\phi \in C:\|\phi\|<\mathbf{B}\}, C=\left\{\phi:[-\tau, 0]_{\mathbf{Z}} \rightarrow \mathbf{R}\right\}$ with $\|\phi\|=$ $\sup _{s \in[-\tau, 0] \mathbf{Z}}|\phi(s)|, f(n, \phi)$ is almost periodic in $n$ uniformly for $\phi \in C_{\mathbf{B}}$ and is continuous in $\phi$, while $x_{n} \in C_{\mathbf{B}}$ is defined as $x_{n}(s)=x(n+s)$ for all $s \in[-\tau, 0]_{\mathbf{Z}}$.

The product system of (1.2) is in the form of

$$
x(n+1)=f\left(n, x_{n}\right), \quad y(n+1)=f\left(n, y_{n}\right) .
$$

A discrete Lyapunov functional of (1.2) is a functional $V: \mathbf{Z}^{+} \times C_{\mathbf{B}} \times C_{\mathbf{B}} \rightarrow \mathbf{R}^{+}$which is continuous in its second and third variables. Define the difference of $V$ along the solution of system (1.2) by

$$
\Delta V_{(1.2)}(n, \phi, \psi)=V\left(n+1, x_{n+1}(n, \phi), y_{n+1}(n, \psi)\right)-V(n, \phi, \psi),
$$

where $(x(n, \phi), y(n, \psi))$ is a solution of system (1.2) through $(n,(\phi, \psi)), \phi, \psi \in C_{\mathbf{B}}$.

Lemma 2.2 [17] Suppose that there exists a Lyapunov functional $V(n, \phi, \psi)$ satisfying the following conditions:

(1) $a(|\phi(0)-\psi(0)|) \leq V(n, \phi, \psi) \leq b(\|\phi-\psi\|)$, where $a, b \in \mathcal{P}$ with $\mathcal{P}=\{a:[0, \infty) \rightarrow[0, \infty) \mid a(0)=0$ and $a(u)$ is continuous, increasing in $u\}$.

(2) $\left|V\left(n, \phi_{1}, \psi_{1}\right)-V\left(n, \phi_{2}, \psi_{2}\right)\right| \leq L\left(\left\|\phi_{1}-\phi_{2}\right\|+\left\|\psi_{1}-\psi_{2}\right\|\right)$, where $L>0$ is a constant.

(3) $\Delta V_{(1.2)}(n, \phi, \psi) \leq-\gamma V(n, \phi, \psi)$, where $0<\gamma<1$ is a constant.

Moreover, if there exists a solution $x(n)$ of (1.2) such that $\left\|x_{n}\right\| \leq B^{*}<B$ for all $n \in \mathbf{Z}^{+}$, then there exists a unique uniformly asymptotically stable almost periodic solution $p(n)$ of (1.2) which satisfies $|p(n)| \leq B^{*}$ for all $n \in \mathbf{I}$. In particular, if $f(n, \phi)$ is periodic of period $\omega$, then (1.2) has a unique uniformly asymptotically stable periodic solution of period $\omega$.

\section{Persistence}

In this section, we establish a persistence result for system (1.2).

Proposition 3.1 Assume that $\left(\mathrm{H}_{1}\right)$ holds. For every solution $\left(x_{1}(n), x_{2}(n), u_{1}(n), u_{2}(n)\right)$ of system (1.2),

$$
\limsup _{n \rightarrow \infty} x_{i}(n)<x_{i}^{*}, \quad \limsup _{n \rightarrow \infty} u_{i}(n)<u_{i}^{*} \quad(i=1,2),
$$

where $x_{1}^{*}=\frac{\exp \left(b^{M}-1\right)}{a^{L}}, x_{2}^{*}=\frac{x_{1}^{*}+\epsilon}{f^{L}} \exp \left(g^{M}-1\right), u_{i}^{*}=\frac{\beta_{i}^{M} x_{i}^{*}}{\alpha_{i}^{L}}(i=1,2)$.

Proof We first present two cases to prove that

$$
\limsup _{n \rightarrow \infty} x_{1}(n)<x_{1}^{*}
$$

Case 1. By the first equation of system (1.2), from $\left(\mathrm{H}_{1}\right)$ and (1.3), we have

$$
\begin{aligned}
x_{1}(n+1) & =x_{1}(n) \exp \left\{b(n)-a(n) x_{1}(n)-\frac{c(n) x_{1}(n) x_{2}(n)}{h^{2}(n) x_{2}^{2}(n)+x_{1}^{2}(n)}-d(n) u_{1}(n)\right\} \\
& <x_{1}(n) \exp \left\{b(n)-a(n) x_{1}(n)-d(n) u_{1}(n)\right\} \\
& =x_{1}(n) \exp \left\{b(n)\left[1-\frac{a(n) x_{1}(n)}{b(n)}-\frac{d(n) u_{1}(n)}{b(n)}\right]\right\} .
\end{aligned}
$$


Then there exists $l_{0} \in \mathbb{N}$ such that $x_{1}\left(l_{0}+1\right) \geq x_{1}\left(l_{0}\right)$. So, $1-\frac{a\left(l_{0}\right) x_{1}\left(l_{0}\right)}{b\left(l_{0}\right)}-\frac{d\left(l_{0}\right) u_{1}\left(l_{0}\right)}{b\left(l_{0}\right)} \geq 0$. Hence, $x_{1}\left(l_{0}\right) \leq b^{L} \leq b^{M}$, and

$$
\begin{aligned}
x_{1}\left(l_{0}+1\right) & <x_{l}\left(l_{0}\right) \exp \left\{b\left(l_{0}\right)-a\left(l_{0}\right) x_{1}\left(l_{0}\right)-d\left(l_{0}\right) u_{1}\left(l_{0}\right)\right\} \\
& \leq x_{1}\left(l_{0}\right) \exp \left\{b^{M}\left[1-\frac{a\left(l_{0}\right) x_{1}\left(l_{0}\right)}{b\left(l_{0}\right)}\right]\right\} \\
& \leq \frac{\exp \left(b^{M}-1\right)}{a^{L}}:=x_{1}^{*} .
\end{aligned}
$$

Here we used $\max _{x \in R} x \exp (r(1-x))=\exp (r-1) / r$ for $r>0$. We claim that $x_{1}(n) \leq x_{1}^{*}$ for $n \geq l_{0}$.

In fact, if there exists an integer $m \geq n_{0}+2$ such that $x_{1}(m)>x_{1}^{*}$, and letting $m_{1}$ be the least integer between $n_{0}$ and $m$ such that $x_{1}(m)=\max _{n_{0} \leq n \leq m-1}\left\{x_{1}(n)\right\}$, then $m_{1} \geq n_{0}+2$ and $x_{1}\left(m_{1}\right)>x_{1}\left(m_{1}-1\right)$, which implies $x_{1}\left(m_{1}\right)<x_{1}^{*}<x_{1}(m)$. This is impossible. The claim is proved.

Case 2. $x_{1}(n) \geq x_{1}(n+1)$ for $n \in \mathbb{N}$. In particular, $\lim _{n \rightarrow \infty} x_{1}(n)$ exists, denoted by $\bar{x}_{1}$. We claim that $\bar{x}_{1}<x_{1}^{*}$. By way of contradiction, assume that $\bar{x}_{1}>x_{1}^{*}$. Taking $\lim _{n \rightarrow \infty}(1-$ $\left.\frac{a(n) x_{1}(n)}{b(n)}-\frac{d(n) u_{1}(n)}{b(n)}\right)=0$. Noting that $b^{M} \leq x_{1}^{*}$, therefore

$$
1-\frac{a(n) x_{1}(n)}{b(n)}-\frac{d(n) u_{1}(n)}{b(n)} \leq 1-\frac{a(n) x_{1}(n)}{b(n)} \leq 1-\frac{\bar{x}_{1}}{b^{M}}<0
$$

for $n \in N$, which is a contradiction. This proves the claim.

Similarly to the above analysis, next we prove $\limsup _{n \rightarrow \infty} x_{2}(n)<x_{2}^{*}$.

Case 1. By the second equation of system (1.2), from $\left(\mathrm{H}_{1}\right)$ and (1.3), we can obtain

$$
\begin{aligned}
x_{2}(n+1) & =x_{2}(n) \exp \left\{g(n)-\frac{f(n) x_{2}(n)}{x_{1}(n)}-p(n) u_{2}(n)\right\} \\
& =x_{1}(n) \exp \left\{g(n)\left[1-\frac{f(n) x_{2}(n)}{g(n) x_{1}(n)}-\frac{p(n) u_{2}(n)}{g(n)}\right]\right\} .
\end{aligned}
$$

Then there exists $l_{0} \in \mathbb{N}$ such that $x_{2}\left(l_{0}+1\right) \geq x_{2}\left(l_{0}\right)$. So, $1-\frac{f\left(l_{0}\right) x_{2}\left(l_{0}\right)}{g\left(l_{0}\right) x_{1}\left(l_{0}\right)}-\frac{p\left(l_{0}\right) u_{2}\left(l_{0}\right)}{g\left(l_{0}\right)} \geq 0$. Hence, $x_{2}\left(l_{0}\right) \leq g^{L} \leq g^{M}$, and

$$
\begin{aligned}
x_{2}\left(l_{0}+1\right) & <x_{2}\left(l_{0}\right) \exp \left[g\left(l_{0}\right)-\frac{f\left(l_{0}\right) x_{2}\left(l_{0}\right)}{g\left(l_{0}\right) x_{1}\left(l_{0}\right)}\right] \\
& \leq x_{2}\left(l_{0}\right) \exp \left\{g^{M}\left[1-\frac{f\left(l_{0}\right) x_{2}\left(l_{0}\right)}{g\left(l_{0}\right) x_{1}\left(l_{0}\right)}\right]\right\} \\
& \leq \frac{x_{1}^{*}+\epsilon}{f^{L}} \exp \left(g^{M}-1\right):=x_{2}^{*} .
\end{aligned}
$$

In fact, if there exists an integer $m \geq n_{0}+2$ such that $x_{2}(m)>x_{2}^{*}$, and letting $m_{2}$ be the least integer between $n_{0}$ and $m$ such that $x_{2}(m)=\max _{n_{0} \leq n \leq m-1}\left\{x_{2}(n)\right\}$, then $m_{1} \geq n_{0}+2$ and $x_{2}\left(m_{2}\right)>x_{2}\left(m_{1}-1\right)$, which implies $x_{2}\left(m_{2}\right)<x_{2}^{*}<x_{2}(m)$. This is impossible. The claim is proved.

Case 2. $x_{2}(n) \geq x_{2}(n+1)$ for $n \in \mathbb{N}$. In particular, $\lim _{n \rightarrow \infty} x_{2}(n)$ exists, denoted by $\bar{x}_{2}$. We claim that $\bar{x}_{2}<x_{2}^{*}$. By way of contradiction, assume that $\bar{x}_{2}>x_{2}^{*}$. Taking $\lim _{n \rightarrow \infty}(1-$ 
$\left.\frac{f(n) x_{2}(n)}{g(n) x_{1}(n)}-\frac{p(n) u_{2}(n)}{g(n)}\right)=0$. Noting that $g^{M} \leq x_{2}^{*}$, therefore

$$
1-\frac{f(n) x_{2}(n)}{g(n) x_{1}(n)}-\frac{p(n) u_{2}(n)}{g(n)} \leq 1-\frac{f(n) x_{2}(n)}{g(n) x_{1}(n)} \leq 1-\frac{f^{L} \bar{x}_{2}}{g^{M}\left(x_{1}^{*}+\epsilon\right)}<0
$$

for $n \in N$, which is a contradiction. This proves the claim.

Similarly, by the third and fourth equations of system (1.2), for all $i=1,2$, we can get

$$
\begin{aligned}
u_{i}(n) & =\prod_{i=0}^{n-1}\left(1-\alpha_{i}(i)\right)\left[u_{i}(0)+\sum_{i=0}^{n-1} \frac{\beta_{i}(i) x_{i}(i)}{\prod_{j=0}^{i}\left(1-\alpha_{i}(j)\right)}\right] \\
& \leq\left(1-\alpha_{i}^{L}\right)^{n}\left[u_{i}(0)+\sum_{i=0}^{n_{0}-1} \frac{\beta_{i}(i) x_{i}(i)}{\prod_{j=0}^{i}\left(1-\alpha_{i}(j)\right)}\right]+\beta_{i}^{M}\left(x_{i}^{*}+\epsilon\right) \sum_{i=n_{0}}^{n-1} \prod_{j=i+1}^{n-1}\left(1-\alpha_{i}(j)\right) \\
& \leq\left(1-\alpha_{i}^{L}\right)^{n}\left[u_{i}(0)+\sum_{i=0}^{n_{0}-1} \frac{\beta_{i}(i) x_{i}(i)}{\prod_{j=0}^{i}\left(1-\alpha_{i}(j)\right)}\right]+\beta_{i}^{M}\left(x_{i}^{*}+\epsilon\right) \sum_{i=n_{0}}^{n-1}\left(1-\alpha_{i}^{L}\right)^{n-i-1} .
\end{aligned}
$$

Since $0<\alpha_{i}^{L}<1$, we can find a positive number $d_{i}$ such that $1-a_{i}^{L}=e^{-d_{i}}$. Using Stolz's theorem, we have

$$
\lim _{n \rightarrow \infty} \sum_{i=n_{0}}^{n-1}\left(1-\alpha_{i}^{L}\right)^{n-i-1}=\lim _{n \rightarrow \infty} \frac{\sum_{i=n_{0}}^{n-1} e^{d_{i}(i+1)}}{e^{d_{i} n}}=\frac{1}{1-e^{-d_{i}}}=\frac{1}{\alpha_{i}^{L}} .
$$

Hence

$$
\lim _{n \rightarrow \infty} \sup u_{i}(n) \leq \frac{\beta_{i}^{M}\left(x_{i}^{*}+\epsilon\right)}{\alpha_{i}^{L}}
$$

By the arbitrariness of $\epsilon, \lim _{n \rightarrow \infty} \sup u_{i}(n) \leq u_{i}^{*}$ is valid. So the proof of Proposition 3.1 is complete.

Proposition 3.2 Assume that $\left(\mathrm{H}_{1}\right)-\left(\mathrm{H}_{3}\right)$ hold, where $x_{i}^{*}$ and $u_{i}^{*}(i=1,2)$ are the same in Proposition 3.1. Then

$$
\liminf _{n \rightarrow \infty} x_{i}(n)>x_{i *}, \quad \liminf _{n \rightarrow \infty} u_{i}(n)>u_{i *}, \quad i=1,2,
$$

where

$$
\begin{aligned}
& x_{1 *}=\Delta_{1} \exp \left\{b^{L}\left[1-\frac{d^{M} u_{1}^{*}}{b^{L}}-\frac{a^{M}+\frac{c^{M}}{h^{M 2} x_{2}^{*}}}{b^{L}}\right]\right\}, \\
& x_{2 *}=\frac{\left(g^{L}-p^{M} u_{2}^{*}\right) x_{1}^{*}}{f^{M}} \exp \left[g^{L}\left(1-\frac{f^{M} x_{2}^{*}}{g^{L} x_{1}^{*}}-\frac{p^{M} u_{2}^{*}}{g^{L}}\right)\right], \quad u_{i *}=\frac{\beta_{i}^{L} x_{i}^{*}}{\alpha_{i}^{M}} .
\end{aligned}
$$

Proof Firstly, we also present two cases to prove that

$$
\liminf _{n \rightarrow \infty} x_{1}(n) \geq x_{1 *} .
$$


For any $\epsilon>0$ which satisfies $\left(b^{L}-d^{M} u_{1}^{*}\right) /\left(a^{M}+c^{M} /\left(h^{M}\right)^{2} x_{2}^{*}\right)>0$ and $\left(g^{L}-p^{M} u_{2}^{*}\right) x_{1}^{*} / f^{M}>0$, according to Proposition 3.1, there exists $n_{0} \in \mathbb{N}$ such that

$$
x_{i}(n) \leq x_{i}^{*}+\epsilon, \quad u_{i}(n) \leq u_{i}^{*}+\epsilon, \quad i=1,2
$$

for $n \geq n_{0}$.

Case 1 . There exists a positive integer $l_{0} \geq n_{0}$ such that $x_{1}\left(l_{0}+1\right) \leq x_{1}\left(l_{0}\right)$. Note that for $n \geq n_{0}$, we have

$$
\begin{aligned}
& x_{1}(n+1) \\
& =x_{1}(n) \exp \left\{b(n)-a(n) x_{1}(n)-\frac{c(n) x_{1}(n) x_{2}(n)}{h^{2}(n) x_{2}^{2}(n)+x_{1}^{2}(n)}-d(n) u_{1}(n)\right\} \\
& >x_{1}(n) \exp \left\{b(n)-a(n) x_{1}(n)-\frac{c(n) x_{1}(n)}{h^{2}(n) x_{2}(n)}-d(n) u_{1}(n)\right\} \\
& =x_{1}(n) \exp \left\{b(n)\left[1-\frac{a(n) x_{1}(n)}{b(n)}-\frac{d(n) u_{1}(n)}{b(n)}-\frac{a(n)+\frac{c(n)}{h^{2}(n) x_{2}(n)}}{b(n)} x_{1}(n)\right]\right\} \\
& \geq x_{1}(n) \exp \left\{b(n)\left[1-\frac{a(n) x_{1}(n)+d(n)\left(u_{1}^{*}+\epsilon\right)}{b(n)}-\frac{a(n)+\frac{c(n)}{h^{2}(n)\left(x_{2}^{*}+\epsilon\right)}}{b(n)} x_{1}(n)\right]\right\} .
\end{aligned}
$$

In particular, with $n=l_{0}$, we obtain

$$
1-\frac{d^{M}\left(u_{1}^{*}+\epsilon\right)}{b\left(l_{0}\right)}-\frac{a^{M}+\frac{c^{M}}{\left(h^{M}\right)^{2}\left(x_{2}^{*}+\epsilon\right)}}{b\left(l_{0}\right)} x_{1}\left(l_{0}\right) \leq 0,
$$

which implies that $x_{1}\left(l_{0}\right) \geq \frac{b^{L}-d^{M}\left(u_{1}^{*}+\epsilon\right)}{a^{M}+\frac{c^{M}}{\left(h^{M}\right)^{2}\left(x_{2}^{*}+\epsilon\right)}}:=\Delta_{1}$. Then

$$
x_{1}\left(l_{0}+1\right)>\Delta_{1} \exp \left\{b^{L}\left[1-\frac{d^{M}\left(u_{1}^{*}+\epsilon\right)}{b^{L}}-\frac{a^{M}+\frac{c^{M}}{h^{M 2}\left(x_{2}^{*}+\epsilon\right)}}{b^{L}}\right]\right\}:=x_{1 \epsilon} .
$$

We claim that $x_{1}(n) \geq x_{1 \epsilon}$ for $n \geq l_{0}$.

By way of contradiction, assume that there exists $p_{0} \geq l_{0}$ such that $x_{1}\left(p_{0}\right)<x_{1 \epsilon}$. Then $p_{0} \geq l_{0}+2$, let $p_{1} \geq l_{0}+2$ be the smallest integer such that $x_{1}\left(p_{1}\right)<x_{1 \epsilon}$. Then $x\left(p_{1}-1\right)<x\left(p_{1}\right)$. The above argument produces that $x_{1}\left(p_{1}\right) \geq x_{1 \epsilon}$, a contradiction. This proves the claim.

Case 2. We assume that $x_{1}(n+1) \geq x_{1}(n)$ for all large $n \in \mathbb{N}$. Then $\lim _{n \rightarrow \infty} x_{1}(n)$ exists, denoted by $\underline{x}_{1}$. We claim that $\underline{x}_{1} \geq \Delta_{1}$. By way of contradiction, assume that $\underline{x}_{1}<\Delta_{1}$. Take

$$
\lim _{n \rightarrow \infty}\left(1-\frac{a(n)+\frac{c(n)}{h^{2}(n) x_{2}(n)}}{b(n)} x_{1}(n)-\frac{d(n) u_{1}(n)}{b(n)}\right)=0
$$

which is a contradiction since

$$
\begin{gathered}
\lim _{n \rightarrow \infty}\left(1-\frac{a(n)+\frac{c(n)}{h^{2}(n) x_{2}(n)}}{b(n)} x_{1}(n)-\frac{d(n) u_{1}(n)}{b(n)}\right) \\
\geq 1-\frac{a^{M}+\frac{c^{M}}{h^{M 2}\left(x_{2}^{*}+\epsilon\right)}}{b(n)} x_{1}(n)-\frac{d^{M}\left(u_{1}^{*}+\epsilon\right)}{b(n)}>0 .
\end{gathered}
$$


Noting that $x_{1}^{*} \geq b^{M} \geq b^{L}$, we see that $\Delta_{1} \geq x_{1 \epsilon}$, and $\lim _{\epsilon \rightarrow 0} x_{1 \epsilon}=x_{1 *}$. We can easily see that $\lim _{n \rightarrow \infty} \inf x_{1}(n) \geq x_{1 *}$ holds.

The same as in the above equality analysis, we will obtain the result from the second equation of system (1.2).

Case 1. By the second equation of system (1.2), $\left(\mathrm{H}_{1}\right)-\left(\mathrm{H}_{3}\right)$ and (1.3), we can obtain

$$
\begin{aligned}
x_{2}(n+1) & =x_{2}(n) \exp \left\{g(n)-\frac{f(n) x_{2}(n)}{x_{1}(n)}-p(n) u_{2}(n)\right\} \\
& >x_{2}(n) \exp \left\{g(n)-\frac{f(n) x_{2}(n)}{x_{1}^{*}+\epsilon}-p(n)\left(u_{2}^{*}+\epsilon\right)\right\} . \\
& =x_{2}(n) \exp \left\{g(n)\left[1-\frac{f(n) x_{2}(n)}{g(n)\left(x_{1}^{*}+\epsilon\right)}-\frac{p(n)\left(u_{2}^{*}+\epsilon\right)}{g(n)}\right]\right\} \\
& \geq x_{2}(n) \exp \left\{g(n)\left[1-\frac{f^{M} x_{2}(n)}{g(n)\left(x_{1}^{*}+\epsilon\right)}-\frac{p^{M}\left(u_{2}^{*}+\epsilon\right)}{g(n)}\right]\right\} .
\end{aligned}
$$

In particular with $n=l_{0}$, we have

$$
1-\frac{f^{M} x_{2}\left(l_{0}\right)}{g\left(l_{0}\right)\left(x_{1}^{*}+\epsilon\right)}-\frac{p^{M}\left(u_{2}^{*}+\epsilon\right)}{g\left(l_{0}\right)} \leq 0,
$$

which implies that

$$
x_{2}\left(l_{0}\right) \geq \frac{\left(g\left(l_{0}\right)-p^{M}\left(u_{2}^{*}+\epsilon\right)\right)\left(x_{1}^{*}+\epsilon\right)}{f\left(l_{0}\right)}:=\Delta_{2} .
$$

Then

$$
x_{2}\left(l_{0}+1\right)>\Delta_{2} \exp \left[g^{L}\left(1-\frac{f^{M}\left(x_{2}^{*}+\epsilon\right)}{g\left(l_{0}\right)\left(x_{1}^{*}+\epsilon\right)}-\frac{p^{M}\left(u_{2}^{*}+\epsilon\right)}{g\left(l_{0}\right)}\right)\right] .
$$

Let $x_{2 \epsilon}=\left(g\left(l_{0}\right)-p^{M}\left(u_{2}^{*}+\epsilon\right)\right)\left(x_{1}^{*}+\epsilon\right) / f\left(l_{0}\right) \exp \left[g^{L}\left(1-f^{M}\left(x_{2}^{*}+\epsilon\right) / g\left(l_{0}\right)\left(x_{1}^{*}+\epsilon\right)-p^{M}\left(u_{2}^{*}+\right.\right.\right.$ $\left.\left.\epsilon) / g\left(l_{0}\right)\right)\right]$. We claim that $x_{2}(n) \geq x_{2 \epsilon}$ for $n \geq n_{0}$.

By way of contradiction, assume that there exists $q_{0} \geq l_{0}$ such that $x_{2}\left(q_{0}\right)<x_{2 \epsilon}$. Then $q_{0} \geq l_{0}+2$, let $q_{0} \geq l_{0}+2$, let $q_{1} \geq l_{0}+2$ be the smallest integer such that $x_{2}\left(q_{0}\right)<x_{2 \epsilon}$. Then $x\left(q_{1}-1\right)<x\left(q_{1}\right)$. The above argument produces that $x_{2}\left(q_{1}\right) \geq x_{2 \epsilon}$, a contradiction. This proves the claim.

Case 2. We assume that $x_{2}(n+1)>x_{2}(n)$ for $n \in \mathbb{N}$. Then $\lim _{n \rightarrow \infty} x_{2}(n)$ exists, denoted by $\underline{x}_{2}$. We claim that

$$
\underline{x}_{2} \geq \frac{\left(g\left(l_{0}\right)-p^{M}\left(u_{2}^{*}+\epsilon\right)\right)\left(x_{1}^{*}+\epsilon\right)}{f\left(l_{0}\right)} .
$$

By way of contradiction, assume that $\underline{x}_{2}<\Delta_{2}$. Take $\lim _{n \rightarrow \infty}\left(1-\frac{f(n) x_{2}(n)}{g(n) x_{1}(n)}-\frac{p(n) u_{2}(n)}{g(n)}\right)=0$, which is a contradiction, since

$$
\liminf _{n \rightarrow \infty}\left(1-\frac{f(n) x_{2}(n)}{g(n) x_{1}(n)}-\frac{p(n) u_{2}(n)}{g(n)}\right) \geq 1-\frac{f^{M} \underline{x}_{2}}{g^{L}\left(x_{1}^{*}+\epsilon\right)}-\frac{p^{M}\left(u_{2}^{*}+\epsilon\right)}{g^{L}}>0 .
$$


Noting that $x_{2}^{*} \geq g^{M} \geq g^{L}$, we see that $\Delta_{2} \geq x_{2 \epsilon}$, and $\lim _{\epsilon \rightarrow 0} x_{2 \epsilon}=x_{2 *}$. We can easily see that $\liminf _{n \rightarrow \infty} x_{2}(n) \geq x_{2 *}$ holds. Thus, for any $\epsilon>0$ small enough, there exists a positive integer $n_{0}$, such that $x_{i}(n) \geq x_{i *}-\epsilon>0$ for $n \geq n_{0}$.

The proof of $\liminf _{n \rightarrow \infty} u_{i}(n)>u_{i *}, i=1,2$, is very similar to that of Proposition 2 in [19]. Here we omit the details.

Now the main result of this section is obtained as follows.

Theorem 3.1 Suppose that assumptions $\left(\mathrm{H}_{1}\right)-\left(\mathrm{H}_{3}\right)$ hold. Then system $(1.2)$ is persistent.

\section{Existence of a unique almost periodic solution}

According to Lemma 2.2, we first prove that there exists a bounded solution of system (1.2) and then construct an adaptive Lyapunov functional for system (1.2).

The next results tells that there exists a bounded solution of system (1.2).

Proposition 4.1 Assume that $\left(\mathrm{H}_{1}\right)-\left(\mathrm{H}_{3}\right)$ hold, then $(S) \neq \emptyset$.

Proof It is now possible to show by an inductive argument that system (1.2) leads to

$$
\left\{\begin{array}{l}
x_{1}(n)=x_{1}(0) \exp \sum_{l=0}^{n-1}\left\{b(l)-a(l) x_{1}(l)-\frac{c(l) x_{1}(l) x_{2}(l)}{h^{2}(l) x_{2}^{2}(l)+x_{1}^{2}(l)}-d(l) u_{1}(l)\right\} \\
x_{2}(n)=x_{2}(0) \exp \sum_{l=0}^{n-1}\left\{g(l)-f(l) \frac{x_{2}(l)}{x_{1}(l)}-p(l) u_{2}(l)\right\} \\
u_{i}(n)=u_{i}(0)-\sum_{l=0}^{n-1}\left\{\alpha_{i}(l) u_{i}(l)-\beta_{i}(l) x_{i}(l)\right\}, \quad i=1,2 .
\end{array}\right.
$$

From Proposition 3.1 and Proposition 3.2, any solution $X(n)=\left(x_{1}(n), x_{2}(n), u_{1}(n), u_{2}(n)\right)$ of system (1.2) with initial condition (1.3) satisfies system (4.1). Hence, for any $\epsilon>0$, there exists $n_{0}$. If $n_{0}$ is sufficiently large, we have

$$
x_{i *}-\epsilon \leq x_{i}(n) \leq x_{i}^{*}+\epsilon, \quad u_{i *}-\epsilon \leq u_{i}(n) \leq u_{i}^{*}+\epsilon, \quad \forall n \geq n_{0}, i=1,2 .
$$

Let $\left\{t_{n}\right\}$ be any integer-valued sequence such that $t_{n} \rightarrow \infty$ as $n \rightarrow \infty$. We claim that there exists a subsequence of $\left\{t_{n}\right\}$, we still denote it by $\left\{t_{n}\right\}$, such that

$$
x_{i}\left(n+t_{n}\right) \rightarrow x_{i}^{*}(n)
$$

uniformly in $n$ on any finite subset $B$ of $Z$ as $n \rightarrow \infty$, where $B=\left\{\alpha_{1}, \alpha_{2}, \ldots, \alpha_{m}\right\}, \alpha_{h} \in \mathbb{Z}$ $(h=1,2, \ldots, m)$ and $m$ is a finite number.

In fact, for any finite subset $B \subset \mathbb{Z}$, when $\alpha$ is large enough, $t_{n}+\alpha_{h}>n_{0}, h=1,2, \ldots, m$. So

$$
x_{i *}-\epsilon \leq x_{i}\left(n+t_{n}\right) \leq x_{i}^{*}+\epsilon, \quad u_{i *}-\epsilon \leq u_{i}\left(n+t_{n}\right) \leq u_{i}^{*}+\epsilon .
$$

That is, $\left\{x_{i}\left(n+t_{n}\right)\right\},\left\{u_{i}\left(n+t_{n}\right)\right\}$ are uniformly bounded for large enough $n$.

Similarly, for $a_{2} \in \mathbb{B}$, we can choose a subsequence $\left\{t_{n}^{2}\right\}$ of $\left\{t_{n}^{1}\right\}$ such that $\left\{x_{i}\left(a_{2}+t_{n}^{2}\right)\right\}$, $\left\{u_{i}\left(a_{2}+t_{n}^{2}\right)\right\}$ uniformly converges on $\mathbb{Z}^{+}$for $n$ large enough.

Repeating this procedure, for $a_{m} \in \mathbb{B}$, we obtain a subsequence $\left\{t_{n}^{m}\right\}$ of $\left\{t_{n}^{m-1}\right\}$ such that $\left\{x_{i}\left(a_{m}+t_{n}^{m}\right)\right\},\left\{u_{i}\left(a_{m}+t_{n}^{m}\right)\right\}$ uniformly converges on $\mathbb{Z}^{+}$for $n$ large enough. 
Now pick the sequence $\left\{t_{n}^{m}\right\}$ which is a subsequence of $\left\{t_{n}\right\}$, we still denote it by $\left\{t_{n}\right\}$, then for all $n \in \mathbb{B}$, we have $x_{i}\left(n+t_{n}\right) \rightarrow x_{i}^{*}(n), u_{i}\left(n+t_{n}\right) \rightarrow u_{i}^{*}(n)$ uniformly in $n \in \mathbb{B}$ as $p \rightarrow \infty$. By the arbitrariness of $\mathbb{B}$, the conclusion is valid.

Since $a(n), b(n), c(n), d(n), h(n), g(n), p(n), f(n), \alpha_{i}(n)$ and $\beta_{i}(n)$ are almost periodic sequences, for the above sequence $\left\{\tau_{p}\right\}, \tau_{p} \rightarrow \infty$ as $p \rightarrow \infty$, there exists a subsequence still denoted by $\left\{\tau_{p}\right\}$ (if necessary, we take a subsequence) such that

$$
\begin{array}{lll}
a\left(n+\tau_{p}\right) \rightarrow a(n), & b\left(n+\tau_{p}\right) \rightarrow b(n), & c\left(n+\tau_{p}\right) \rightarrow c(n), \\
d\left(n+\tau_{p}\right) \rightarrow d(n), & g\left(n+\tau_{p}\right) \rightarrow g(n), & f\left(n+\tau_{p}\right) \rightarrow f(n), \\
p\left(n+\tau_{p}\right) \rightarrow p(n), & \alpha_{i}\left(n+\tau_{p}\right) \rightarrow \alpha_{i}(n), & \beta_{i}\left(n+\tau_{p}\right) \rightarrow \beta_{i}(n), \quad i=1,2,
\end{array}
$$

as $p \rightarrow \infty$ uniformly on $\mathbb{Z}^{+}$. For any $\sigma \in \mathbb{Z}$, we can assume that $\tau_{p}+\sigma \geq n_{0}$ for $p$ large enough. Let $n \geq 0$ and $n \in \mathbb{Z}^{+}$, an inductive argument of system (1.2) from $\tau_{p}+\sigma$ to $n+$ $\tau_{p}+\sigma$ leads to

$$
\left\{\begin{array}{l}
x_{1}\left(n+\tau_{p}+\sigma\right)=x_{1}\left(\tau_{p}+\sigma\right) \exp \sum_{l=\tau_{p}+\sigma}^{n+\tau_{p}+\sigma-1}\left\{b(l)-a(l) x_{1}(l)-\frac{c(l) x_{1}(l) x_{2}(l)}{h^{2}(l) x_{2}^{2}(l)+x_{1}^{2}(l)}-d(l) u_{1}(l)\right\} \\
x_{2}\left(n+\tau_{p}+\sigma\right)=x_{2}\left(\tau_{p}+\sigma\right) \exp \sum_{l=\tau_{p}+\sigma}^{n+\tau_{p}+\sigma-1}\left\{g(l)-f(l) \frac{x_{2}(l)}{x_{1}(l)}-p(l) u_{2}(l)\right\} \\
u_{i}\left(n+\tau_{p}+\sigma\right)=u_{i}\left(\tau_{p}+\sigma\right)-\sum_{l=\tau_{p}+\sigma}^{n+\tau_{p}+\sigma-1}\left\{\alpha_{i}(l) u_{i}(l)-\beta_{i}(l) x_{i}(l)\right\}
\end{array}\right.
$$

Then, for $i=1,2$, we have

$$
\left\{\begin{aligned}
x_{1}\left(n+\tau_{p}+\sigma\right)= & x_{1}\left(\tau_{p}+\sigma\right) \exp \sum_{l=\sigma}^{n+\sigma-1}\left\{b\left(l+\tau_{p}\right)-a\left(l+\tau_{p}\right) x_{1}\left(l+\tau_{p}\right)\right. \\
& \left.\times \frac{c\left(l+\tau_{p}\right) x_{1}\left(l+\tau_{p}\right) x_{2}\left(l+\tau_{p}\right)}{h^{2}\left(l+\tau_{p}\right) x_{2}^{2}\left(l+\tau_{p}\right)+x_{1}^{2}\left(l+\tau_{p}\right)}-d\left(l+\tau_{p}\right) u_{1}\left(l+\tau_{p}\right)\right\}, \\
x_{2}\left(n+\tau_{p}+\sigma\right)= & x_{2}\left(\tau_{p}+\sigma\right) \exp \sum_{l=\sigma}^{n+\sigma-1}\left\{g\left(l+\tau_{p}\right)-f\left(l+\tau_{p}\right) \frac{x_{2}\left(l+\tau_{p}\right)}{x_{1}\left(l+\tau_{p}\right)}\right. \\
& \left.-p\left(l+\tau_{p}\right) u_{2}\left(l+\tau_{p}\right)\right\}, \\
u_{i}\left(n+\tau_{p}+\sigma\right)= & u_{i}\left(\tau_{p}+\sigma\right)-\sum_{l=\sigma}^{n+\sigma-1}\left\{\alpha_{i}\left(l+\tau_{p}\right) u_{i}\left(l+\tau_{p}\right)-\beta_{i}\left(l+\tau_{p}\right) x_{i}\left(l+\tau_{p}\right)\right\} .
\end{aligned}\right.
$$

Let $p \rightarrow \infty$, for any $n \geq 0$,

$$
\left\{\begin{array}{l}
x_{1}^{*}(n+\sigma)=x_{1}^{*}(\sigma) \exp \sum_{l=\sigma}^{n+\sigma-1}\left\{b(l)-a(l) x_{1}^{*}(l)-\frac{c(l) x_{1}^{*}(l) x_{2}^{*}}{h^{2}(l) x_{2}^{* 2}(l)+x_{1}^{* 2}(l)}-d(l) u_{1}^{*}(l)\right\}, \\
x_{2}^{*}(n+\sigma)=x_{2}^{*}(\sigma) \sum_{l=\sigma}^{n+\sigma-1} \exp \left\{g(l)-f(l) \frac{x_{2}^{*}(l)}{x_{1}^{*}(l)}-p(l) u_{2}^{*}(l)\right\} \\
u_{i}^{*}(n+\sigma)=u_{i}^{*}(\sigma)-\sum_{l=\sigma}^{n+\sigma-1}\left\{\alpha_{i}(l) u_{i}^{*}(l)-\beta_{i}(l) x_{i}^{*}(l)\right\} .
\end{array}\right.
$$

By the arbitrariness of $\sigma, X^{*}=\left(x_{1}^{*}(n), x_{2}^{*}(n), u_{1}^{*}(n), u_{2}^{*}(n)\right)$ is a solution of system (1.2) on $\mathbb{Z}^{+}$. It is clear that $0<x_{i *} \leq x_{i}^{*}(n) \leq x_{i}^{*}, 0<u_{i *} \leq u_{i}^{*}(n) \leq u_{i}^{*}$, for all $n \in \mathbb{Z}^{+}, i=1,2$. So $\Omega \neq \emptyset$. Proposition 4.1 is valid.

The main results of the following theorem concern the existence of a uniformly asymptotically stable almost periodic sequence solution of system (1.2).

Theorem 4.1 Assume that $\left(\mathrm{H}_{1}\right)-\left(\mathrm{H}_{3}\right)$ hold. Suppose further that $\left(\mathrm{H}_{4}\right): 0<\Theta<1$, here $\Theta=$ $\min \left\{\Theta_{1}, \Theta_{2}, \Theta_{3}, \Theta_{4}\right\}$, where

$$
\begin{aligned}
\Theta_{1}= & 2 a^{L} x_{1 *}-\frac{4 c^{M 2} x_{1}^{* 4} x_{2}^{* 2}}{\left[h^{L 2} x_{2 *}^{2}+x_{1 *}^{2}\right]^{4}}-d^{M}-\frac{4 c^{M} x_{1}^{* 2} x_{2}^{*}}{\left[h^{L 2} x_{2 *}^{2}+x_{1 *}^{2}\right]^{2}} \\
& -\frac{c^{M} x_{2}^{*}\left(h^{M 2} x_{2}^{* 2}+x_{1}^{* 2}\right)}{\left[h^{L 2} x_{2 *}^{2}+x_{1 *}^{2}\right]^{2}}-a^{M} d^{M} x_{1}^{*}-a^{M} x_{1}^{*}
\end{aligned}
$$




$$
\begin{aligned}
& -\frac{8 a^{M} c^{M 2} x_{1}^{* 4} x_{2}^{* 2}}{\left[h^{L 2} x_{2 *}^{2}+x_{1 *}^{2}\right]^{4}}-\frac{8 d^{M} c^{M 2} x_{1}^{* 4} x_{2}^{* 2}}{\left[h^{L 2} x_{2 *}^{2}+x_{1 *}^{2}\right]^{4}}-\frac{f^{M 2} x_{1}^{* 2} x_{2}^{* 2}}{x_{1 *}^{4}}-\frac{f^{M} x_{1}^{*} x_{2}^{*}}{x_{1 *}^{2}} \\
& -\frac{2 p^{M} f^{M 2} x_{1}^{* 2} x_{2}^{* 2}}{x_{1 *}^{4}}-\beta_{1}^{M} x_{1}^{*}-\left(1-\alpha_{1}^{L}\right) x_{1}^{*} \beta_{1}^{M}-a^{M 2} x_{1}^{* 2}, \\
\Theta_{2}= & \frac{2 f^{L} x_{1 *} x_{2 *}}{x_{1}^{* 2}}-\frac{c^{M 2} x_{2}^{* 2}\left(h^{M 2} x_{2}^{* 2}+x_{1}^{* 2}\right)^{2}}{\left[h^{L 2} x_{2 *}^{2}+x_{1 *}^{2}\right]^{4}}-\frac{c^{M} x_{2}^{*}\left(h^{M 2} x_{2}^{* 2}+x_{1}^{* 2}\right)}{\left[h^{L 2} x_{2 *}^{2}+x_{1 *}^{2}\right]^{2}}-p^{M} \\
& -\frac{2 a^{M} c^{M 2} x_{2}^{* 2}\left(h^{M 2} x_{2}^{* 2}+x_{1}^{* 2}\right)^{2}}{\left[h^{L 2} x_{2 *}^{2}+x_{1 *}^{2}\right]^{4}}-\frac{2 d^{M} c^{M 2} x_{2}^{* 2}\left(h^{M 2} x_{2}^{* 2}+x_{1}^{* 2}\right)^{2}}{\left[h^{L 2} x_{2 *}^{2}+x_{1 *}^{2}\right]^{4}}-\frac{f^{M 2} x_{1}^{* 2} x_{2}^{* 2}}{x_{1 *}^{4}} \\
& -\frac{2 f^{M} x_{1}^{*} x_{2}^{*}}{x_{1 *}^{2}}-\frac{2 p^{M} f^{M 2} x_{1}^{* 2} x_{2}^{* 2}}{x_{1 *}^{4}}-\beta_{2}^{M} x_{2}^{*}-\left(1-\alpha_{2}^{L}\right) x_{2}^{*} \beta_{2}^{M}, \\
\Theta_{3}= & 2 \alpha_{1}^{L}-d^{M}-d^{M 2}-a^{M} d^{M}-\alpha_{1}^{L 2}-\left(1-\alpha_{1}^{L}\right) \beta_{1}^{M}
\end{aligned}
$$

and

$$
\Theta_{4}=2 \alpha_{2}^{L}-p^{M 2}-2 p^{M}-\alpha_{2}^{L 2}-\left(1-\alpha_{2}^{L}\right) \beta_{2}^{M},
$$

then there exists a unique uniformly asymptotically stable almost periodic solution $X(n)=$ $\left(x_{1}(n), x_{2}(n), u_{1}(n), u_{2}(n)\right)$ of system (1.2) which is bounded by $\Omega$ for all $n \in \mathbf{Z}^{+}$.

Proof Let $p_{i}(n)=\ln x_{i}(n)$. From (1.2), we have

$$
\begin{aligned}
p_{1}(n+1)= & p_{1}(n)+b(n)-a(n) e^{p_{1}(n)} \\
& -c(n) \frac{e^{p_{2}(n)}}{h^{2}(n) e^{2 p_{2}(n)}+e^{2 p_{1}(n)}}-d(n) u_{1}(n), \\
p_{2}(n+1)= & p_{2}(n)+g(n)-f(n) \frac{e^{p_{2}(n)}}{e^{p_{1}(n)}}-p(n) u_{2}(n), \\
\Delta u_{i}(n)= & -\alpha_{i}(n) u_{i}(n)+\beta_{i}(n) e^{p_{i}(n)},
\end{aligned}
$$

where $i=1$,2. From Proposition 4.1, we know that system (4.8) has a bounded solution $Y(n)=\left(p_{1}(n), p_{2}(n), u_{1}(n), u_{2}(n)\right)$ satisfying

$$
\ln x_{i *} \leq p_{i}(n) \leq \ln x_{i}^{*}, \quad u_{i *} \leq u_{i}(n) \leq u_{i}^{*}, \quad i=1,2, n \in \mathbf{Z}^{+} .
$$

Hence, $\left|p_{i}(n)\right| \leq A_{i},\left|u_{i}(n)\right| \leq B_{i}$, where $A_{i}=\max \left\{\left|\ln x_{i *}\right|, \ln x_{i}^{*}\right\}, B_{i}=\max \left\{u_{i_{*}}, u_{i}^{*}\right\}, i=1,2$.

For $(X, U) \in R^{2+2}$, we define the norm $\|(X, U)\|=\sum_{i=1}^{2}\left|x_{i}\right|+\sum_{i=1}^{2}\left|u_{i}\right|$.

Consider the product system of system (4.8)

$$
\left\{\begin{array}{l}
p_{1}(n+1)=p_{1}(n)+b(n)-a(n) e^{p_{1}(n)}-\frac{c(n) e^{p_{2}(n)}}{h^{2}(n) e^{2 p_{2}(n)}+e^{2 p_{1}(n)}}-d(n) u_{1}(n), \\
p_{2}(n+1)=p_{2}(n)+g(n)-f(n) \frac{e^{p_{2}(n)}}{e^{p_{1}(n)}}-p(n) u_{2}(n), \\
\Delta u_{i}(n)=-\alpha_{i}(n) u_{i}(n)+\beta_{i}(n) e^{p_{i}(n)} \\
q_{1}(n+1)=q_{1}(n)+b(n)-a(n) e^{q_{1}(n)}-\frac{c(n) e^{q_{2}(n)}}{h^{2}(n) e^{2 q_{2}(n)}+e^{2 q_{1}(n)}}-d(n) \omega_{1}(n), \\
q_{2}(n+1)=q_{2}(n)+g(n)-f(n) \frac{e^{q_{2}(n)}}{e^{q_{1}(n)}}-p(n) \omega_{2}(n), \\
\Delta \omega_{i}(n)=-\alpha_{i}(n) \omega_{i}(n)+\beta_{i}(n) e^{q_{i}(n)} .
\end{array}\right.
$$


Suppose that $Z=\left(p_{1}(n), p_{2}(n), u_{1}(n), u_{2}(n)\right), W=\left(q_{1}(n), q_{2}(n), \omega_{1}(n), \omega_{2}(n)\right)$ are any two solutions of system (4.10) defined on $Z^{+} \times S^{*} \times S^{*}$, then $\|Z\| \leq B,\|W\| \leq B$, where

$$
\begin{aligned}
B= & \sum_{i=1}^{2}\left\{A_{i}+B_{i}\right\}, \\
S^{*}= & \left\{\left(p_{1}(n), p_{2}(n), u_{1}(n), u_{2}(n)\right) \mid \ln x_{i *} \leq p_{i}(n) \leq \ln x_{i}^{*},\right. \\
& \left.u_{i *} \leq u_{i}(n) \leq u_{i}^{*}, i=1,2, n \in Z^{+}\right\} .
\end{aligned}
$$

Consider the Lyapunov function defined on $Z^{+} \times S^{*} \times S^{*}$ as follows:

$$
V(n, Z, W)=\sum_{i=1}^{2}\left\{\left(p_{i}(n)-q_{i}(n)\right)^{2}+\left(u_{i}(n)-\omega_{i}(n)\right)^{2}\right\} .
$$

It is easy to see that the norm $\|Z-W\|=\sum_{i=1}^{2}\left\{\left|p_{i}(n)-q_{i}(n)\right|+\left|u_{i}(n)-\omega_{i}(n)\right|\right\}$ and the norm $\|Z-W\|_{*}=\left\{\sum_{i=1}^{2}\left\{\left(p_{i}(n)-q_{i}(n)\right)^{2}+\left(u_{i}(n)-\omega_{i}(n)\right)^{2}\right\}\right\}^{1 / 2}$ are equivalent, that is, there exist two constants $C_{1}>0, C_{2}>0$ such that

$$
C_{1}\|Z-W\| \leq\|Z-W\|_{*} \leq C_{2}\|Z-W\|
$$

then

$$
\left(C_{1}\|Z-W\|\right)^{2} \leq\|Z-W\|_{*} \leq\left(C_{2}\|Z-W\|\right)^{2} .
$$

Let $a \in C\left(R^{+}, R^{+}\right), a(x)=C_{1}^{2} x^{2}, b \in C\left(R^{+}, R^{+}\right), b(x)=C_{2}^{2} x^{2}$, thus condition (1) in Lemma 2.2 is satisfied.

In addition,

$$
\begin{aligned}
|V(n, Z, W)-V(n, \tilde{Z}, \tilde{W})| \\
=\mid \sum_{i=1}^{2}\left\{\left(p_{i}(n)-q_{i}(n)\right)^{2}+\left(u_{i}(n)-\omega_{i}(n)\right)^{2}\right\} \\
\quad-\sum_{i=1}^{2}\left\{\left(\tilde{p}_{i}(n)-\tilde{q}_{i}(n)\right)^{2}+\left(\tilde{u}_{i}(n)-\tilde{\omega}_{i}(n)\right)^{2}\right\} \mid \\
\leq \sum_{i=1}^{2}\left|\left(p_{i}(n)-q_{i}(n)\right)^{2}+\left(u_{i}(n)-\omega_{i}(n)\right)^{2}\right|+\sum_{i=1}^{2}\left|\left(\tilde{p}_{i}(n)-\tilde{q}_{i}(n)\right)^{2}+\left(\tilde{u}_{i}(n)-\tilde{\omega}_{i}(n)\right)^{2}\right| \\
=\sum_{i=1}^{2}\left\{\left|\left(p_{i}(n)-q_{i}(n)\right)+\left(\tilde{p}_{i}(n)-\tilde{q}_{i}(n)\right)\right|\left|\left(p_{i}(n)-q_{i}(n)\right)-\left(\tilde{p}_{i}(n)-\tilde{q}_{i}(n)\right)\right|\right\} \\
\quad \times \sum_{i=1}^{2}\left\{\left|\left(u_{i}(n)-\omega_{i}(n)\right)+\left(\tilde{u}_{i}(n)-\tilde{\omega}_{i}(n)\right)\right|\left\|\left(u_{i}(n)-\omega_{i}(n)\right)-\left(\tilde{u}_{i}(n)-\tilde{\omega}_{i}(n)\right)\right\|\right\} \\
\leq \sum_{i=1}^{2}\left\{\left(\left|p_{i}(n)\right|+\left|q_{i}(n)\right|+\left|\tilde{p}_{i}(n)\right|+\left|\tilde{q}_{i}(n)\right|\right)\left(\left|p_{i}(n)-\tilde{p}_{i}(n)\right|+\left|q_{i}(n)-\tilde{q}_{i}(n)\right|\right)\right\} \\
\quad \times \sum_{i=1}^{2}\left\{\left(\left|u_{i}(n)\right|+\left|\omega_{i}(n)\right|+\left|\tilde{u}_{i}(n)\right|+\left|\tilde{\omega}_{i}(n)\right|\right)\left(\left|u_{i}(n)-\tilde{u}_{i}(n)\right|+\left|\omega_{i}(n)-\tilde{\omega}_{i}(n)\right|\right)\right\}
\end{aligned}
$$




$$
\begin{aligned}
& \leq L\left\{\sum_{i=1}^{2}\left\{\left|p_{i}(n)-\tilde{p}_{i}(n)\right|+\left|u_{i}(n)-\tilde{u}_{i}(n)\right|\right\}+\sum_{i=1}^{2}\left\{\left|q_{i}(n)-\tilde{q}_{i}(n)\right|+\left|\omega_{i}(n)-\tilde{\omega}_{i}(n)\right|\right\}\right\} \\
& =L\{\|Z-\tilde{Z}\|+\|W-\tilde{W}\|\},
\end{aligned}
$$

where $L=\max \left\{A_{i}, B_{i}\right\}(i=1,2)$. Hence condition (2) of Lemma 2.2 is satisfied.

Finally, calculating $\Delta V$ of $V(n)$ along the solutions of (4.10), we can obtain

$$
\begin{aligned}
\Delta V_{(4.10)}(n, Z, W)= & V(n+1, Z, W)-V(n, Z, W) \\
= & \sum_{i=1}^{2}\left\{\left[p_{i}(n+1)-q_{i}(n+1)\right]^{2}+\left(u_{i}(n+1)-\omega_{i}(n+1)\right)^{2}\right\} \\
& -\sum_{i=1}^{2}\left\{\left[p_{i}(n)-q_{i}(n)\right]^{2}+\left[u_{i}(n)-\omega_{i}(n)\right]^{2}\right\} \\
= & \sum_{i=1}^{2}\left\{\left(p_{i}(n+1)-q_{i}(n+1)\right)^{2}-\left(p_{i}(n)-q_{i}(n)\right)^{2}\right. \\
& \left.+\left(u_{i}(n+1)-\omega_{i}(n+1)\right)^{2}-\left(u_{i}(n)-\omega_{i}(n)\right)^{2}\right\} \\
= & \sum_{i=1}^{2}\left\{\left[p_{i}(n+1)-q_{i}(n+1)\right]^{2}-\left(p_{i}(n)-q_{i}(n)\right)^{2}\right. \\
& +\left[\left(1-\alpha_{i}(n)\right)\left(u_{i}(n)-\omega_{i}(n)\right)+\beta_{i}(n)\left(e^{p_{i}(n)}-e^{q_{i}(n)}\right)\right]^{2} \\
& \left.-\left(u_{i}(n)-\omega_{i}(n)\right)^{2}\right\} .
\end{aligned}
$$

In view of system (4.1) and using the mean value theorem, we get

$$
e^{p_{i}(n)}-e^{q_{i}(n)}=\xi_{i}(n)\left(p_{i}(n)-q_{i}(n)\right), \quad i=1,2,
$$

where $\xi_{i}(n)$ lies between $e^{p_{i}(n)}$ and $e^{q_{i}(n)}, i=1,2$,

$$
\begin{aligned}
& {\left[p_{1}(n+1)-q_{1}(n+1)\right]^{2}} \\
& =\left[\left(p_{1}(n)-q_{1}(n)\right)-a(n)\left[e^{p_{1}(n)}-e^{q_{1}(n)}\right]-d(n)\left[u_{1}(n)-\omega_{1}(n)\right]\right. \\
& \left.-c(n)\left(\frac{e^{p_{2}(n)}}{h^{2}(n) e^{2 p_{2}(n)}+e^{2 p_{1}(n)}}-\frac{e^{q_{2}(n)}}{h^{2}(n) e^{2 q_{2}(n)}+e^{2 q_{1}(n)}}\right)\right]^{2} \\
& =\left[p_{1}(n)-q_{1}(n)\right]^{2}+a^{2}(n)\left[e^{p_{1}(n)}-e^{q_{1}(n)}\right]^{2}+d^{2}(n)\left[u_{1}(n)-\omega_{1}(n)\right]^{2} \\
& +c^{2}(n)\left[\frac{e^{p_{2}(n)}}{h^{2}(n) e^{2 p_{2}(n)}+e^{2 p_{1}(n)}}-\frac{e^{q_{2}(n)}}{h^{2}(n) e^{2 q_{2}(n)}+e^{2 q_{1}(n)}}\right]^{2} \\
& -2 a(n)\left[p_{1}(n)-q_{1}(n)\right]\left[e^{p_{1}(n)}-e^{q_{1}(n)}\right]-2 d(n)\left[p_{1}(n)-q_{1}(n)\right]\left[u_{1}(n)-\omega_{1}(n)\right] \\
& -2 c(n)\left[p_{1}(n)-q_{1}(n)\right]\left[\frac{e^{p_{2}(n)}}{h^{2}(n) e^{2 p_{2}(n)}+e^{2 p_{1}(n)}}-\frac{e^{q_{2}(n)}}{h^{2}(n) e^{2 q_{2}(n)}+e^{2 q_{1}(n)}}\right] \\
& +2 a(n) d(n)\left[e^{p_{1}(n)}-e^{q_{1}(n)}\right]\left[u_{1}(n)-\omega_{1}(n)\right] \\
& +2 a(n) c(n)\left[e^{p_{1}(n)}-e^{q_{1}(n)}\right]\left[\frac{e^{p_{2}(n)}}{h^{2}(n) e^{2 p_{2}(n)}+e^{2 p_{1}(n)}}-\frac{e^{q_{2}(n)}}{h^{2}(n) e^{2 q_{2}(n)}+e^{2 q_{1}(n)}}\right]
\end{aligned}
$$




$$
\begin{aligned}
& +2 c(n) d(n)\left[u_{1}(n)-\omega_{1}(n)\right]\left[\frac{e^{p_{2}(n)}}{h^{2}(n) e^{2 p_{2}(n)}+e^{2 p_{1}(n)}}-\frac{e^{q_{2}(n)}}{h^{2}(n) e^{2 q_{2}(n)}+e^{2 q_{1}(n)}}\right] \\
\leq & {\left[p_{1}(n)-q_{1}(n)\right]^{2}+a^{M 2} x_{1}^{* 2}\left[p_{1}(n)-q_{1}(n)\right]^{2}+d^{M 2}\left[u_{1}(n)-\omega_{1}(n)\right]^{2} } \\
& +W_{1}^{2}(n)+W_{2}(n)+W_{3}(n)+W_{4}(n)+W_{5}(n)+W_{6}(n),
\end{aligned}
$$

where

$$
\begin{aligned}
& W_{1}(n)=c(n)\left[\frac{e^{p_{2}(n)}}{h^{2}(n) e^{2 p_{2}(n)}+e^{2 p_{1}(n)}}-\frac{e^{q_{2}(n)}}{h^{2}(n) e^{2 q_{2}(n)}+e^{2 q_{1}(n)}}\right] \\
& =c(n) \frac{h^{2}(n) e^{p_{2}(n)} e^{2 q_{2}(n)}+e^{p_{2}(n)} e^{2 q_{1}(n)}-h^{2}(n) e^{q_{2}(n)} e^{2 p_{2}(n)}-e^{q_{2}(n)} e^{2 p_{1}(n)}}{\left[h^{2}(n) e^{2 p_{2}(n)}+e^{2 p_{1}(n)}\right]\left[h^{2}(n) e^{2 q_{2}(n)}+e^{2 q_{1}(n)}\right]} \\
& \leq \frac{c^{M}\left(h^{M 2} x_{2}^{* 2}+x_{1}^{* 2}\right)\left|e^{p_{2}(n)}-e^{q_{2}(n)}\right|+2 c^{M} x_{1}^{*} x_{2}^{*}\left|e^{p_{1}(n)}-e^{q_{1}(n)}\right|}{\left[h^{l 2} x_{2 *}^{2}+x_{1 *}^{2}\right]^{2}} \\
& \leq \frac{2 c^{M} x_{1}^{* 2} x_{2}^{*}}{\left[h^{l 2} x_{2 *}^{2}+x_{1 *}^{2}\right]^{2}}\left|p_{1}(n)-q_{1}(n)\right|+\frac{c^{M} x_{2}^{*}\left(h^{M 2} x_{2}^{* 2}+x_{1}^{* 2}\right)}{\left[h^{l 2} x_{2 *}^{2}+x_{1 *}^{2}\right]^{2}}\left|p_{2}(n)-q_{2}(n)\right|, \\
& W_{2}(n)=-2 a(n)\left[p_{1}(n)-q_{1}(n)\right]\left[e^{p_{1}(n)}-e^{q_{1}(n)}\right]-2 d(n)\left[p_{1}(n)-q_{1}(n)\right]\left[u_{1}(n)-\omega_{1}(n)\right] \\
& \leq-2 a^{l} x_{1 *}\left[p_{1}(n)-q_{1}(n)\right]^{2}+d^{M}\left[p_{1}(n)-q_{1}(n)\right]^{2}+d^{M}\left[u_{1}(n)-\omega_{1}(n)\right]^{2}, \\
& W_{3}(n)=-2\left[p_{1}(n)-q_{1}(n)\right] W_{1}(n) \\
& =-2 c(n)\left[p_{1}(n)-q_{1}(n)\right]\left[\frac{e^{p_{2}(n)}}{h^{2}(n) e^{2 p_{2}(n)}+e^{2 p_{1}(n)}}-\frac{e^{q_{2}(n)}}{h^{2}(n) e^{2 q_{2}(n)}+e^{2 q_{1}(n)}}\right] \\
& \leq 2 c(n)\left|\left[p_{1}(n)-q_{1}(n)\right]\right|\left|\frac{e^{p_{2}(n)}}{h^{2}(n) e^{2 p_{2}(n)}+e^{2 p_{1}(n)}}-\frac{e^{q_{2}(n)}}{h^{2}(n) e^{2 q_{2}(n)}+e^{2 q_{1}(n)}}\right| \\
& \leq \frac{4 c^{M} x_{1}^{* 2} x_{2}^{*}}{\left[h^{l 2} x_{2 *}^{2}+x_{1 *}^{2}\right]^{2}}\left[p_{1}(n)-q_{1}(n)\right]^{2} \\
& +\frac{2 c^{M} x_{2}^{*}\left(h^{M 2} x_{2}^{* 2}+x_{1}^{* 2}\right)}{\left[h^{l 2} x_{2 *}^{2}+x_{1 *}^{2}\right]^{2}}\left|p_{1}(n)-q_{1}(n)\right|\left|p_{2}(n)-q_{2}(n)\right| \\
& \leq \frac{4 c^{M} x_{1}^{* 2} x_{2}^{*}}{\left[h^{l 2} x_{2 *}^{2}+x_{1 *}^{2}\right]^{2}}\left[p_{1}(n)-q_{1}(n)\right]^{2} \\
& +\frac{c^{M} x_{2}^{*}\left(h^{M 2} x_{2}^{* 2}+x_{1}^{* 2}\right)}{\left[h^{l 2} x_{2 *}^{2}+x_{1 *}^{2}\right]^{2}}\left[p_{1}(n)-q_{1}(n)\right]^{2}+\frac{c^{M} x_{2}^{*}\left(h^{M 2} x_{2}^{* 2}+x_{1}^{* 2}\right)}{\left[h^{l 2} x_{2 *}^{2}+x_{1 *}^{2}\right]^{2}}\left[p_{2}(n)-q_{2}(n)\right]^{2}, \\
& W_{4}(n)=2 a(n) d(n)\left[e^{p_{1}(n)}-e^{q_{1}(n)}\right]\left[u_{1}(n)-\omega_{1}(n)\right] \\
& \leq a^{M} d^{M} x_{1}^{*}\left[p_{1}(n)-q_{1}(n)\right]^{2}+a^{M} d^{M}\left[u_{1}(n)-\omega_{1}(n)\right]^{2}, \\
& W_{5}(n)=2 a(n) c(n)\left[e^{p_{1}(n)}-e^{q_{1}(n)}\right]\left[\frac{e^{p_{2}(n)}}{h^{2}(n) e^{2 p_{2}(n)}+e^{2 p_{1}(n)}}-\frac{e^{q_{2}(n)}}{h^{2}(n) e^{2 q_{2}(n)}+e^{2 q_{1}(n)}}\right] \\
& \leq a^{M}\left[e^{p_{1}(n)}-e^{q_{1}(n)}\right]^{2}+a^{M} W_{1}^{2} \\
& \leq a^{M} x_{1}^{*}\left[p_{1}(n)-q_{1}(n)\right]^{2} \\
& +a^{M}\left[\frac{2 c^{M} x_{1}^{* 2} x_{2}^{*}}{\left[h^{l 2} x_{2 *}^{2}+x_{1 *}^{2}\right]^{2}}\left|p_{1}(n)-q_{1}(n)\right|+\frac{c^{M} x_{2}^{*}\left(h^{M 2} x_{2}^{* 2}+x_{1}^{* 2}\right)}{\left[h^{l 2} x_{2 *}^{2}+x_{1 *}^{2}\right]^{2}}\left|p_{2}(n)-q_{2}(n)\right|\right]^{2} \\
& \leq a^{M} x_{1}^{*}\left[p_{1}(n)-q_{1}(n)\right]^{2}
\end{aligned}
$$




$$
\begin{aligned}
& +\frac{8 a^{M} c^{M 2} x_{1}^{* 4} x_{2}^{* 2}}{\left[h^{l 2} x_{2 *}^{2}+x_{1 *}^{2}\right]^{4}}\left|p_{1}(n)-q_{1}(n)\right|^{2} \\
& +\frac{2 a^{M} c^{M 2} x_{2}^{* 2}\left(h^{M 2} x_{2}^{* 2}+x_{1}^{* 2}\right)^{2}}{\left[h^{l 2} x_{2 *}^{2}+x_{1 *}^{2}\right]^{4}}\left|p_{2}(n)-q_{2}(n)\right|^{2}
\end{aligned}
$$

and

$$
\begin{aligned}
W_{6}(n)= & 2 c(n) d(n)\left[u_{1}(n)-\omega_{1}(n)\right]\left[\frac{e^{p_{2}(n)}}{h^{2}(n) e^{2 p_{2}(n)}+e^{2 p_{1}(n)}}-\frac{e^{q_{2}(n)}}{h^{2}(n) e^{2 q_{2}(n)}+e^{2 q_{1}(n)}}\right] \\
\leq & d^{M}\left[u_{1}(n)-\omega_{1}(n)\right]^{2}+\frac{8 d^{M} c^{M 2} x_{1}^{* 4} x_{2}^{* 2}}{\left[h^{l 2} x_{2 *}^{2}+x_{1 *}^{2}\right]^{4}}\left|p_{1}(n)-q_{1}(n)\right|^{2} \\
& +\frac{2 d^{M} c^{M 2} x_{2}^{* 2}\left(h^{M 2} x_{2}^{* 2}+x_{1}^{* 2}\right)^{2}}{\left[h^{l 2} x_{2 *}^{2}+x_{1 *}^{2}\right]^{4}}\left|p_{2}(n)-q_{2}(n)\right|^{2}
\end{aligned}
$$

Similarly, we also have

$$
\begin{aligned}
{\left[p_{2}(n+1)-q_{2}(n+1)\right]^{2} } & \left(\left[p_{2}(n)-q_{2}(n)\right]-f(n)\left[\frac{e^{p_{2}(n)}}{e^{p_{1}(n)}}-\frac{e^{q_{2}(n)}}{e^{q_{1}(n)}}\right]-p(n)\left[u_{2}(n)-\omega_{2}(n)\right]\right)^{2} \\
= & {\left[p_{2}(n)-q_{2}(n)\right]^{2}+f^{2}(n)\left[\frac{e^{p_{2}(n)}}{e^{p_{1}(n)}}-\frac{e^{q_{2}(n)}}{e^{q_{1}(n)}}\right]^{2}+p^{2}(n)\left[u_{2}(n)-\omega_{2}(n)\right]^{2} } \\
& -2 f(n)\left[p_{2}(n)-q_{2}(n)\right]\left[\frac{e^{p_{2}(n)}}{e^{p_{1}(n)}}-\frac{e^{q_{2}(n)}}{e^{q_{1}(n)}}\right]-2 p(n)\left[p_{2}(n)-q_{2}(n)\right]\left[u_{2}(n)-\omega_{2}(n)\right] \\
& +2 f(n) p(n)\left[\frac{e^{p_{2}(n)}}{e^{p_{1}(n)}}-\frac{e^{q_{2}(n)}}{e^{q_{1}(n)}}\right]\left[u_{2}(n)-\omega_{2}(n)\right] \\
\leq & {\left[p_{2}(n)-q_{2}(n)\right]^{2}+p^{M 2}\left[u_{2}(n)-\omega_{2}(n)\right]^{2}+K_{1}^{2}(n)+K_{2}(n)+K_{3}(n)+K_{4}(n), }
\end{aligned}
$$

where

$$
\begin{aligned}
K_{1}(n)= & f(n)\left[\frac{e^{p_{2}(n)}}{e^{p_{1}(n)}}-\frac{e^{q_{2}(n)}}{e^{q_{1}(n)}}\right] \\
= & f(n) \frac{e^{p_{2}(n)} e^{q_{1}(n)}-e^{p_{1}(n)} e^{q_{2}(n)}}{e^{p_{1}(n)} e^{q_{1}(n)}} \\
\leq & \frac{f^{M} x_{1}^{*} x_{2}^{*}}{x_{1 *}^{2}}\left|p_{1}(n)-q_{1}(n)\right|+\frac{f^{M} x_{1}^{*} x_{2}^{*}}{x_{1 *}^{2}}\left|p_{2}(n)-q_{2}(n)\right|, \\
K_{2}(n)= & -2 f(n)\left[p_{2}(n)-q_{2}(n)\right]\left[\frac{e^{p_{2}(n)}}{e^{p_{1}(n)}}-\frac{e^{q_{2}(n)}}{e^{q_{1}(n)}}\right] \\
= & -2 f(n)\left[p_{2}(n)-q_{2}(n)\right] \frac{e^{q_{1}(n)}\left(e^{p_{2}(n)}-e^{q_{2}(n)}\right)}{e^{p_{1}(n)} e^{q_{1}(n)}} \\
& -2 f(n)\left[p_{2}(n)-q_{2}(n)\right] \frac{e^{q_{2}(n)}\left(e^{q_{1}(n)}-e^{p_{1}(n)}\right)}{e^{p_{1}(n)} e^{q_{1}(n)}} \\
\leq & -\frac{2 f^{l} x_{1 *} x_{2 *}}{x_{1}^{* 2}}\left[p_{2}(n)-q_{2}(n)\right]^{2}+\frac{2 f^{M} x_{1}^{*} x_{2}^{*}}{x_{1 *}^{2}}\left|\left[p_{1}(n)-q_{1}(n)\right]\left[p_{2}(n)-q_{2}(n)\right]\right| \\
\leq & -\frac{2 f^{l} x_{1 *} x_{2 *}}{x_{1}^{* 2}}\left[p_{2}(n)-q_{2}(n)\right]^{2}+\frac{f^{M} x_{1}^{*} x_{2}^{*}}{x_{1 *}^{2}}\left(\left[p_{1}(n)-q_{1}(n)\right]^{2}+\left[p_{2}(n)-q_{2}(n)\right]^{2}\right),
\end{aligned}
$$




$$
\begin{aligned}
K_{3}(n) & =-2 p(n)\left[p_{2}(n)-q_{2}(n)\right]\left[u_{2}(n)-\omega_{2}(n)\right] \\
& =p^{M}\left(\left[p_{2}(n)-q_{2}(n)\right]^{2}+\left[u_{2}(n)-\omega_{2}(n)\right]^{2}\right)
\end{aligned}
$$

and

$$
\begin{aligned}
K_{4}(n)= & 2 f(n) p(n)\left[\frac{e^{p_{2}(n)}}{e^{p_{1}(n)}}-\frac{e^{q_{2}(n)}}{e^{q_{1}(n)}}\right]\left[u_{2}(n)-\omega_{2}(n)\right] \\
= & p^{M} K_{1}^{2}(n)+p^{M}\left[u_{2}(n)-\omega_{2}(n)\right]^{2} \\
\leq & \frac{2 p^{M} f^{M 2} x_{1}^{* 2} x_{2}^{* 2}}{x_{1 *}^{4}}\left|p_{1}(n)-q_{1}(n)\right|^{2} \\
& +\frac{2 p^{M} f^{M 2} x_{1}^{* 2} x_{2}^{* 2}}{x_{1 *}^{4}}\left|p_{2}(n)-q_{2}(n)\right|^{2}+p^{M}\left[u_{2}(n)-\omega_{2}(n)\right]^{2} .
\end{aligned}
$$

From system (4.10), we also obtain

$$
\begin{aligned}
& {\left[u_{i}(n+1)-\omega_{i}(n+1)\right]^{2}-\left[u_{i}(n)-\omega_{i}(n)\right]^{2}} \\
& \quad=\left[\left(1-\alpha_{i}(n)\right)^{2}-1\right]\left(u_{i}(n)-\omega_{i}(n)\right)^{2}+\beta_{i}^{2}(n)\left(e^{p_{i}(n)}-e^{q_{i}(n)}\right)^{2} \\
& \quad+2 \beta_{i}(n)\left(1-\alpha_{i}(n)\right)\left(u_{i}(n)-\omega_{i}(n)\right)\left(e^{p_{i}(n)}-e^{q_{i}(n)}\right) \\
& \quad \leq\left(\alpha_{i}^{l 2}-2 \alpha_{i}^{l}\right)\left(u_{i}(n)-\omega_{i}(n)\right)^{2}+\beta_{i}^{M} x_{i}^{*}\left(p_{i}(n)-q_{i}(n)\right)^{2}+H_{i}(n),
\end{aligned}
$$

where

$$
\begin{aligned}
H_{i}(n) & =2 \beta_{i}(n)\left(1-\alpha_{i}(n)\right)\left(u_{i}(n)-\omega_{i}(n)\right)\left(e^{p_{i}(n)}-e^{q_{i}(n)}\right) \\
& \leq\left(1-\alpha_{i}^{l}\right) x_{i}^{*} \beta_{i}^{M}\left[p_{i}(n)-q_{i}(n)\right]^{2}+\left(1-\alpha_{i}^{l}\right) \beta_{i}^{M}\left[u_{i}(n)-\omega_{i}(n)\right]^{2} .
\end{aligned}
$$

From (4.16), (4.17), (4.18) and (4.19), we have

$$
\begin{aligned}
\Delta V_{(4.10)}(n) \leq & {\left[a^{M 2} x_{1}^{* 2}+\frac{4 c^{M 2} x_{1}^{* 4} x_{2}^{* 2}}{\left[h^{l 2} x_{2 *}^{2}+x_{1 *}^{2}\right]^{4}}-2 a^{l} x_{1 *}+d^{M}+\frac{4 c^{M} x_{1}^{* 2} x_{2}^{*}}{\left[h^{l 2} x_{2 *}^{2}+x_{1 *}^{2}\right]^{2}}\right.} \\
& +\frac{c^{M} x_{2}^{*}\left(h^{M 2} x_{2}^{* 2}+x_{1}^{* 2}\right)}{\left[h^{l 2} x_{2 *}^{2}+x_{1 *}^{2}\right]^{2}}+a^{M} d^{M} x_{1}^{*}+a^{M} x_{1}^{*}+\frac{8 a^{M} c^{M 2} x_{1}^{* 4} x_{2}^{* 2}}{\left[h^{l 2} x_{2 *}^{2}+x_{1 *}^{2}\right]^{4}} \\
& +\frac{8 d^{M} c^{M 2} x_{1}^{* 4} x_{2}^{* 2}}{\left[h^{l 2} x_{2 *}^{2}+x_{1 *}^{2}\right]^{4}}+\frac{f^{M 2} x_{1}^{* 2} x_{2}^{* 2}}{x_{1 *}^{4}}+\frac{f^{M} x_{1}^{*} x_{2}^{*}}{x_{1 *}^{2}} \\
& \left.+\frac{2 p^{M} f^{M 2} x_{1}^{* 2} x_{2}^{* 2}}{x_{1 *}^{4}}+\beta_{1}^{M} x_{1}^{*}+\left(1-\alpha_{1}^{l}\right) x_{1}^{*} \beta_{1}^{M}\right]\left[p_{1}(n)-q_{1}(n)\right]^{2} \\
& +\left[\frac{c^{M 2} x_{2}^{* 2}\left(h^{M 2} x_{2}^{* 2}+x_{1}^{* 2}\right)^{2}}{\left[h^{l 2} x_{2 *}^{2}+x_{1 *}^{2}\right]^{4}}+\frac{c^{M} x_{2}^{*}\left(h^{M 2} x_{2}^{* 2}+x_{1}^{* 2}\right)}{\left[h^{l 2} x_{2 *}^{2}+x_{1 *}^{2}\right]^{2}}\right. \\
& +\frac{2 a^{M} c^{M 2} x_{2}^{* 2}\left(h^{M 2} x_{2}^{* 2}+x_{1}^{* 2}\right)^{2}}{\left[h^{l 2} x_{2 *}^{2}+x_{1 *}^{2}\right]^{4}} \\
& +\frac{2 d^{M} c^{M 2} x_{2}^{* 2}\left(h^{M 2} x_{2}^{* 2}+x_{1}^{* 2}\right)^{2}}{\left[h^{l 2} x_{2 *}^{2}+x_{1 *}^{2}\right]^{4}}+\frac{f^{M 2} x_{1}^{* 2} x_{2}^{* 2}}{x_{1 *}^{4}}+p^{M}-\frac{2 f^{l} x_{1 *} x_{2 *}}{x_{1}^{* 2}} \\
& \left.+\frac{2 f^{M} x_{1}^{*} x_{2}^{*}}{x_{1 *}^{2}}+\frac{2 p^{M} f^{M 2} x_{1}^{* 2} x_{2}^{* 2}}{x_{1 *}^{4}}+\beta_{2}^{M} x_{2}^{*}+\left(1-\alpha_{2}^{l}\right) x_{2}^{*} \beta_{2}^{M}\right]\left[p_{2}(n)-q_{2}(n)\right]^{2}
\end{aligned}
$$




$$
\begin{aligned}
& +\left[d^{M}+d^{M 2}+a^{M} d^{M}+\left(\alpha_{1}^{l 2}-2 \alpha_{1}^{l}\right)+\left(1-\alpha_{1}^{l}\right) \beta_{1}^{M}\right]\left[u_{1}(n)-\omega_{1}(n)\right]^{2} \\
& +\left[p^{M 2}+2 p^{M}+\left(\alpha_{2}^{l 2}-2 \alpha_{2}^{l}\right)+\left(1-\alpha_{2}^{l}\right) \beta_{2}^{M}\right]\left[u_{2}(n)-\omega_{2}(n)\right]^{2} \\
= & -\left[2 a^{l} x_{1 *}-\frac{4 c^{M 2} x_{1}^{* 4} x_{2}^{* 2}}{\left[h^{l 2} x_{2 *}^{2}+x_{1 *}^{2}\right]^{4}}-d^{M}-\frac{4 c^{M} x_{1}^{* 2} x_{2}^{*}}{\left[h^{l 2} x_{2 *}^{2}+x_{1 *}^{2}\right]^{2}}\right. \\
& -\frac{c^{M} x_{2}^{*}\left(h^{M 2} x_{2}^{* 2}+x_{1}^{* 2}\right)}{\left[h^{l 2} x_{2 *}^{2}+x_{1 *}^{2}\right]^{2}}-a^{M} d^{M} x_{1}^{*}-a^{M} x_{1}^{*}-\frac{8 a^{M} c^{M 2} x_{1}^{* 4} x_{2}^{* 2}}{\left[h^{l 2} x_{2 *}^{2}+x_{1 *}^{2}\right]^{4}} \\
& -\frac{8 d^{M} c^{M 2} x_{1}^{* 4} x_{2}^{* 2}}{\left[h^{l 2} x_{2 *}^{2}+x_{1 *}^{2}\right]^{4}}-\frac{f^{M 2} x_{1}^{* 2} x_{2}^{* 2}}{x_{1 *}^{4}}-\frac{f^{M} x_{1}^{*} x_{2}^{*}}{x_{1 *}^{2}} \\
& \left.-\frac{2 p^{M} f^{M 2} x_{1}^{* 2} x_{2}^{* 2}}{x_{1 *}^{4}}-\beta_{1}^{M} x_{1}^{*}-\left(1-\alpha_{1}^{l}\right) x_{1}^{*} \beta_{1}^{M}-a^{M 2} x_{1}^{* 2}\right]\left[p_{1}(n)-q_{1}(n)\right]^{2} \\
& -\left[\frac{2 f^{l} x_{1 *} x_{2 *}}{x_{1}^{* 2}}-\frac{c^{M 2} x_{2}^{* 2}\left(h^{M 2} x_{2}^{* 2}+x_{1}^{* 2}\right)^{2}}{\left[h^{l 2} x_{2 *}^{2}+x_{1 *}^{2}\right]^{4}}-\frac{c^{M} x_{2}^{*}\left(h^{M 2} x_{2}^{* 2}+x_{1}^{* 2}\right)}{\left[h^{l 2} x_{2 *}^{2}+x_{1 *}^{2}\right]^{2}}-p^{M}\right. \\
& -\frac{2 a^{M} c^{M 2} x_{2}^{* 2}\left(h^{M 2} x_{2}^{* 2}+x_{1}^{* 2}\right)^{2}}{\left[h^{l 2} x_{2 *}^{2}+x_{1 *}^{2}\right]^{4}}-\frac{2 d^{M} c^{M 2} x_{2}^{* 2}\left(h^{M 2} x_{2}^{* 2}+x_{1}^{* 2}\right)^{2}}{\left[h^{l 2} x_{2 *}^{2}+x_{1 *}^{2}\right]^{4}}-\frac{f^{M 2} x_{1}^{* 2} x_{2}^{* 2}}{x_{1 *}^{4}} \\
& \left.-\frac{2 f^{M} x_{1}^{*} x_{2}^{*}}{x_{1 *}^{2}}-\frac{2 p^{M} f^{M 2} x_{1}^{* 2} x_{2}^{* 2}}{x_{1 *}^{4}}-\beta_{2}^{M} x_{2}^{*}-\left(1-\alpha_{2}^{l}\right) x_{2}^{*} \beta_{2}^{M}\right]\left(p_{2}(n)-q_{2}(n)\right)^{2} \\
& -\left[2 \alpha_{1}^{l}-d^{M}-d^{M 2}-a^{M} d^{M}-\alpha_{1}^{l 2}-\left(1-\alpha_{1}^{l}\right) \beta_{1}^{M}\right]\left[u_{1}(n)-\omega_{1}(n)\right]^{2} \\
& -\left[2 \alpha_{2}^{l}-p^{M 2}-2 p^{M}-\alpha_{2}^{l 2}-\left(1-\alpha_{2}^{l}\right) \beta_{2}^{M}\right]\left[u_{2}(n)-\omega_{2}(n)\right]^{2} \\
\leq & -\Theta \sum_{i=1}^{2}\left\{\left(p_{i}(n)-q_{i}(n)\right)^{2}+\left(u_{i}(n)-\omega_{i}(n)\right)^{2}\right\} \\
= & -\Theta V(n, X, Y),
\end{aligned}
$$

where $\Theta=\min \left\{\Theta_{1}, \Theta_{2}, \Theta_{3}, \Theta_{4}\right\}$. That is, there exists a positive constant $0<\Theta<1$ such that $\Delta_{(4.10)}(n, X, Y) \leq-\Theta V(n, X, Y)$. From $0<\Theta<1$, condition (3) of Lemma 2.2 is satisfied. So, from Lemma 2.2, there exists a unique uniformly asymptotically stable almost periodic solution $X(n)=\left(x_{1}(n), x_{2}(n), u_{1}(n), u_{2}(n)\right)$ of system (4.10) which is bounded by $S^{*}$ for all $n \in Z^{+}$, which means that there exists a unique uniformly asymptotically stable almost periodic solution $X(n)=\left(x_{1}(n), x_{2}(n), u_{1}(n), u_{2}(n)\right)$ of system $(1.2)$ which is bounded by $\Omega$ for all $n \in Z^{+}$. This completes the proof.

\section{An example}

In this section, we present an example to illustrate the feasibility of our results.

Example 5.1 Consider the following discrete ratio-dependent Leslie model:

$$
\left\{\begin{array}{l}
x_{1}(n+1)=x_{1}(n) \exp \left\{b(n)-a(n) x_{1}(n)-\frac{c(n) x_{1}(n) x_{2}(n)}{h^{2}(n) x_{2}^{2}(n)+x_{1}^{2}(n)}-d(n) u_{1}(n)\right\} \\
x_{2}(n+1)=x_{2}(n) \exp \left\{g(n)-f(n) \frac{x_{2}(n)}{x_{1}(n)}-p(n) u_{2}(n)\right\} \\
\Delta u_{i}(n)=-\alpha_{i}(n) u_{i}(n)+\beta_{i}(n) x_{i}(n), \quad i=1,2
\end{array}\right.
$$

where $b(n)=2.65+0.65 \sin n, a(n)=0.455+0.045 \sin n, c(n)=0.002+0.001 \sin n, h(n)=$ $5.1+3.9 \sin n, d(n)=0.002, g(n)=0.5, f(n)=4.175-0.225 \cos n, p(n)=0.00005-$ $0.00004 \cos n, \alpha_{1}(n)=8+4 \sin n, \beta_{1}(n)=1.49+1.23 \sin n, \alpha_{2}(n)=4-0.9 \cos n, \beta_{2}(n)=$ 
$0.92+0.72 \cos n$. Then system (5.1) is persistent and has a unique uniformly asymptotically stable almost periodic sequence solution.

Proof It is easy to see that $\{a(n)\},\{b(n)\},\{c(n)\},\{d(n)\},\{h(n)\},\{g(n)\},\{f(n)\},\{p(n)\},\left\{\alpha_{i}(n)\right\}$ and $\left\{\beta_{i}(n)\right\}$ for $i=1,2$ are bounded nonnegative almost periodic sequences. By calculation of Mathematica software, we get

$$
\begin{aligned}
& x_{1}^{*}=24.3273, \quad x_{1 *}=21.9004, \\
& x_{2}^{*}=3.7355, \quad x_{2 *}=0.105256, \\
& u_{1}^{*}=16.5425, \quad u_{2}^{*}=1.9762, \\
& \Theta_{1} \approx 0.372768, \quad \Theta_{2} \approx 0.78929, \\
& \Theta_{3} \approx 0.156996, \quad \Theta_{4} \approx 0.03382 .
\end{aligned}
$$

Then $g^{L}-p^{M} u_{2}^{*}=0.5-0.00009 \times 1.9762=0.49982>0, b^{L}-d^{M} u_{1}^{*}=2-0.002 \times 16.5425=$ $1.96691>0$ and $0<\Theta=\min \left\{\Theta_{1}, \Theta_{2}, \Theta_{3}, \Theta_{4}\right\}=0.03382<1$. So we can see that all the conditions of Theorem 4.1 hold. According to Theorem 4.1, system (5.1) has a unique uniformly asymptotically stable almost periodic solution which is bounded by $\Omega$ for all $n \in Z^{+}$.

\section{Competing interests}

The author declares that they have no competing interests.

\section{Acknowledgements}

The author thanks the anonymous referees for their careful reading of this manuscript, and for their valuable comments and suggestions. This work is supported by Yunnan Province Education Department Scientific Research Fund Project (No. 2012Z065) and the Young Teachers Program of Yuxi Normal University.

Received: 25 March 2014 Accepted: 11 July 2014 Published: 04 Aug 2014

\section{References}

1. Leslie, PH: Some further notes on the use of matrices in population mathematics. Biometrika 35, $213-245$ (1948)

2. Li, YK, Zhang, TW: Permanence and almost periodic sequence solution for a discrete delay logistic equation with feedback control. Nonlinear Anal., Real World Appl. 12(3), 1850-1864 (2011)

3. Wang, Q, Zhou, J, Wang, ZJ, Ding, MM, Zhang, HY: Existence and attractivity of a periodic solution for a ratio-dependent Leslie system with feedback controls. Nonlinear Anal., Real World Appl. 12, 24-33 (2011)

4. Chen, FD: Permanence in nonautonomous multi-species predator-prey system with feedback controls. Comput. Math. Appl. 173, 694-709 (2006)

5. Chen, $\mathrm{F}, \mathrm{CaO}, \mathrm{XH}$ : Existence of almost periodic solution in a ratio-dependent Leslie system with feedback controls. J. Math. Anal. Appl. 341, 1399-1412 (2008)

6. Chen, LJ, Chen, FD: Global stability of a Leslie-Gower predator-prey model with feedback controls. Appl. Math. Lett. 22, 1330-1334 (2009)

7. Weng, $P$, Jiang, D: Existence and global stability of positive periodic solution of $n$-species Lotka-Volterra competition system with feedback controls and deviating arguments. Far East J. Math. Sci. 7(1), 45-65 (2002)

8. Zhang, TW, Li, YK, Ye, Y: Persistence and almost periodic solutions for a discrete fishing model with feedback control. Commun. Nonlinear Sci. Numer. Simul. 16, 1564-1573 (2011)

9. Wang, Z, Li, YK: Almost periodic solutions of a discrete mutualism model with feedback controls. Discrete Dyn. Nat. Soc. 2010, Article ID 286031 (2010)

10. Guo, HJ, Song, XY: An impulsive predator-prey system with modified Leslie-Gower and Holling type II schemes. Chaos Solitons Fractals 36, 1320-1331 (2008)

11. Chen, YP, Liu, ZJ, Haque, M: Analysis of a Leslie-Gower-type prey-predator model with periodic impulsive perturbations. Commun. Nonlinear Sci. Numer. Simul. 14, 3412-3423 (2009)

12. Nindjin, AF, Aziz-Alaoui, MA, Cadivel, M: Analysis of a predator-prey model with modified Leslie-Gower and Holling-type II schemes with time delay. Nonlinear Anal., Real World Appl. 7, 1104-1118 (2006)

13. Song, YL, Yuan, SL, Zhang, JM: Bifurcation analysis in the delayed Leslie-Gower predator-prey system. Appl. Math. Model. 33, 4049-4061 (2006)

14. Gakkhar, S, Singh, B: Dynamics of modified Leslie-Gower-type prey-predator model with seasonally varying parameters. Chaos Solitons Fractals 27, 1239-1255 (2006) 
15. $\mathrm{Du}, \mathrm{YH}$, Peng, R, Wang, MX: Effect of a protection zone in the diffusive Leslie predator-prey model. J. Differ. Equ. 246, 3932-3956 (2009)

16. Aziz-Alaoui, MA: Study of a Leslie-Gower-type tritrophic population model. Chaos Solitons Fractals 14, 1275-1293 (2002)

17. Zhang, SN, Zheng, G: Almost periodic solutions of delay difference systems. Appl. Math. Comput. 131, 497-516 (2002)

18. Zhang, TW, Gan, XR: Almost periodic solutions for a discrete fishing model with feedback control and time delays. Commun. Nonlinear Sci. Numer. Simul. 19, 150-163 (2014)

19. Murray, JD: Mathematical Biology. Biomathematics, vol. 19. Springer, Berlin (1989)

10.1186/1687-1847-2014-214

Cite this article as: Li: Persistence and almost periodic solutions for a discrete ratio-dependent Leslie system with feedback control. Advances in Difference Equations 2014, 2014:214

Submit your manuscript to a SpringerOpen ${ }^{\circ}$ journal and benefit from:

- Convenient online submission

- Rigorous peer review

- Immediate publication on acceptance

- Open access: articles freely available online

- High visibility within the field

- Retaining the copyright to your article

Submit your next manuscript at $\gg$ springeropen.com 